\title{
Risk Factors for Gastrointestinal Injuries in Acute Coronary Syndrome Patients with Double Antiplatelet Therapy in One-Year Follow-Up
}

\author{
Ling Zhong1, Xin Chen"1, Xihua Qiu², Xueli Zhang1, Hua Shao', Yamin Liu1* ${ }^{*}$, Jing Xiong”\# \\ ${ }^{1}$ Division of Pharmacy, Zhongda Hospital, Southeast University, Nanjing, China \\ ${ }^{2}$ Department of Material Supply, Sinopec Jinling Company, Nanjing, China \\ ${ }^{3}$ Division of Pharmacology, Nanjing Medical University, Nanjing, China \\ Email: *Yamin.Liu@ucsf.edu, *liuyamincn@hotmail.com, "xiong.jing@njmu.edu.cn
}

How to cite this paper: Zhong, L., Chen, X., Qiu, X.H., Zhang, X.L., Shao, H., Liu, Y.M. and Xiong, J. (2018) Risk Factors for Gastrointestinal Injuries in Acute Coronary Syndrome Patients with Double Antiplatelet Therapy in One-Year Follow-Up. World Journal of Cardiovascular Diseases, $\mathbf{8}$, 467-480.

https://doi.org/10.4236/wjcd.2018.810046

Received: September 5, 2018

Accepted: October 12, 2018

Published: October 15, 2018

Copyright $(0) 2018$ by authors and Scientific Research Publishing Inc. This work is licensed under the Creative Commons Attribution International License (CC BY 4.0).

http://creativecommons.org/licenses/by/4.0/

\begin{abstract}
Background: The goal is to determine the incidence of symptomatic gastrointestinal (GI) injuries in acute coronary syndrome (ACS) patients receiving double antiplatelet therapy (DAPT). The risk factors for serious GI complications are also evaluated. Methods: 603 eligible patients from the Department of Cardiology at Zhongda Hospital between January 2014 and August 2015 were enrolled and the occurrence of GI injuries within one year assessed. The risk factors for serious GI complications were identified using cox regression analysis. Results: After one-year follow-up, 108 (17.9\%) out of 603 patients developed symptomatic GI injuries: 22 (3.65\%) with serious GI complications and $86(14.2 \%)$ with GI symptoms. Drinking habit (95\% CI: 1.512 - 8.796; $\mathrm{P}=0.004)$ and previous peptic injury (95\% CI: 2.307 - 18.080; $\mathrm{P}$ $=0.001)$ are independent predictors of serious GI complications, while proton pump inhibitor (PPI) was protective (95\% CI: $0.120-0.699 ; \mathrm{P}=0.006$ ) per cox regression analysis. Additionally, GI injuries of both serious GI complications and GI symptoms peaked in the first three months. Conclusions: Symptomatic GI injuries were relatively common in ACS patients with DAPT, especially in the first three months. Previous peptic injury and drinking habit were significant independent risk factors for serious GI complications, while PPI played a protective role in ACS with DAPT.
\end{abstract}

\section{Keywords}

Acute Coronary Syndrome, Double Antiplatelet Therapy, Proton Pump Inhibitor, Serious Gastrointestinal Complications, Symptomatic Gastrointestinal Injury, Risk Factors 


\section{Introduction}

Acute coronary syndrome (ACS) is a clinical syndrome comprised of ST segment elevation myocardial infarction (STEMI), non-ST segment elevation myocardial infarction (NSTEMI), and unstable angina (UA). It is characterized by ruptured coronary plaque, platelet activation and aggregation, ischemic stress, and/or myocardial injury [1] [2]. Several decades of investigation and discovery regarding the pathophysiology of plaque rupture and thrombus formation and the clinical benefits of inhibiting platelet activation and aggregation [3] [4] [5] [6] have led to double antiplatelet therapy (clopidogrel combined with low-dose aspirin, DAPT) as a standard antiplatelet therapy [7] [8].

However, long-time treatment with aspirin and/or clopidogrel has an adverse effect on peptic, ranging from ulcers to hemorrhage and perforation. One study has shown that aspirin alone increases the risk of upper GI bleeding (UGIB) by 1.55 times, and combined with clopidogrel by 1.86 times [9]. Additionally, ACS - a critical condition-is associated with stress-related mucosal injury that increases the risk of GI injury, and complications such as heart failure and arrhythmia can cause insufficient blood circulation to the digestive system and constriction of the mesenteric artery, also leading to GI injuries [10]. Among which, symptomatic GI injury would reduce the patient's compliance with antiplatelet drugs, thereby increasing the risk of cardiovascular events. As such, it is essential to assess the incidence and identify risk factors in order to develop strategies that reduce symptomatic GI injury for ACS patients receiving DAPT.

Currently, only a few studies have reported on the incidence of GI bleeding in patients with DAPT [11] [12]. Further, these studies focused on GI bleeding, especially UGIB, which is only one possible outcome of GI injury. It is important to evaluate the impact of DAPT on various types of GI injury including serious GI complications and GI symptoms over a long period. In this study, we aim to assess the occurrence of more types of GI injury at 12-month follow-up. Additionally, we identify the risk factors for serious GI complications in ACS patients who received DAPT, which always lead to discontinuation of antiplatelet drugs.

\section{Methods}

\subsection{Study Population}

The study protocol was approved by the Independent Ethics Committee of Zhongda Hospital, Southeast University, Jiangsu Province, China, and was conducted in accordance with the guidelines of the Declaration of Helsinki. Patients diagnosed as ACS (STEMI, NSTEMI, UA) who received DAPT (aspirin $100 \mathrm{mg}$ daily and clopidogrel $75 \mathrm{mg}$ daily after loading dose) were recruited from the Department of Cardiology at Zhongda Hospital from January 2014 to August 2015. Further inclusion criteria were platelet counts of $100-300 \times 10^{9} / 1$ and hemoglobin $\geq 10 \mathrm{~g} / \mathrm{dl}$, and age $\geq 18$ years. Patients were excluded if in the past month they received double therapy or PPI or had uncomfortable peptic symptom before screening; had severe hemorrhagic diseases, including cerebral he- 
morrhage and severe trauma; had other serious organ diseases, including malignancies, serious liver and kidney failure, or respiratory failure; or received glucocorticoid.

\subsection{Procedure}

The flow chart is shown in Figure 1. According to inclusion criteria, a total of 645 ACS patients who received DAPT were screened. Of these candidates, 12 patients experienced other serious diseases in organs (including malignancies, severe liver failure, and respiratory failure) and 30 had incomplete data or were lost to follow-up during the study period. Ultimately, 603 eligible patients were enrolled. During the hospital, demographics characteristic of patients was collected by a case report form (CRF). When discharged, notices were given to the patients to remind them to inform the doctor if GI symptoms happen. GI symptoms include dyspepsia, diarrhea, abdominal pains, heartburn, acid regurgitation, sucking sensations in the epigastrium, nausea and vomiting, abdominal distension, eructation, which were listed on the notice. In addition, telephone follow-up was also conducted every month to reduce the impact of poor compliance. GI examinations such as endoscopy, colonoscopy, ultrasonography were carried out in all patients who suffered uncomfortable GI symptoms in time. The GI events and use of antiplatelet drugs were followed up after discharge for one year. Finally, the results of diagnosis, occurrence time came from phone interview, outpatient clinic record review, and readmission records review, were added in the CRF.

\subsection{Clinical Outcomes}

The endpoint was the occurrence of symptomatic GI injury in the patients with

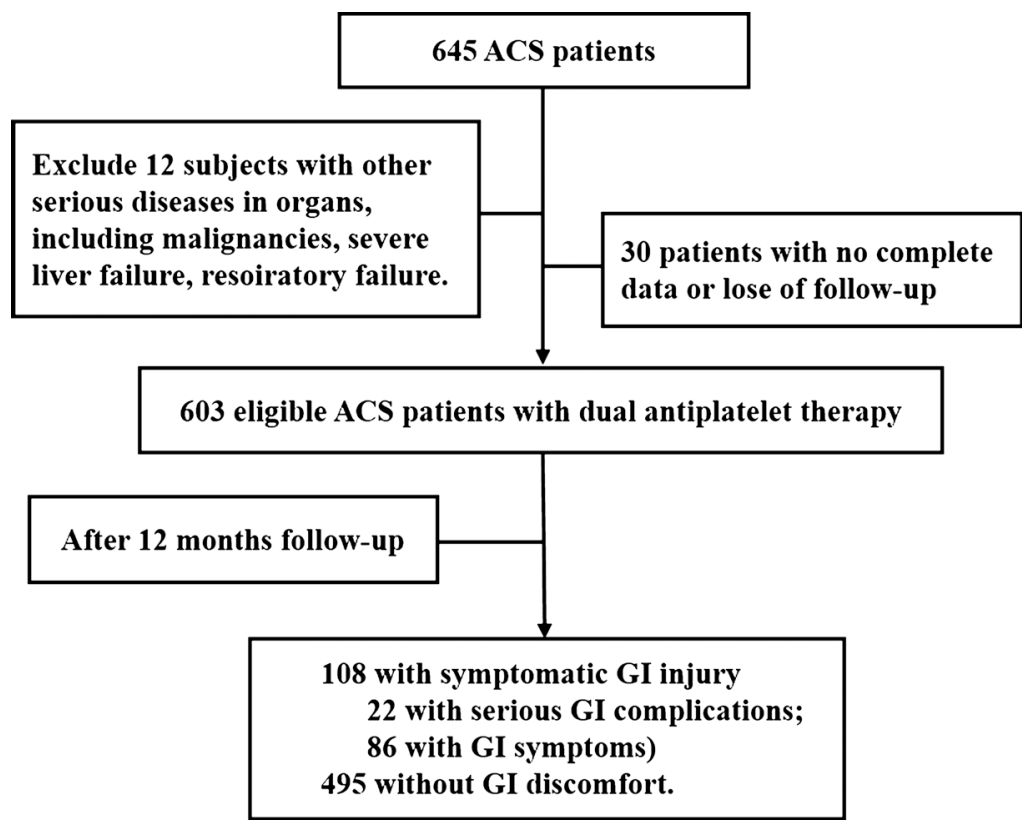

Figure 1. Flowchart of patient disposition and follow-up. 
ACS receiving DAPT, including serious GI complications and GI symptoms according to Brown's classification [1]. Serious GI complications [13], are defined based on the diagnosis as upper GI bleeding, perforation, ulcers, pyloric obstruction and even related death. GI symptoms is defined as a damage discovered when patients complained dyspepsia, diarrhea, abdominal pains, heartburn, acid regurgitation, sucking sensations in the epigastrium, nausea and vomiting, abdominal distension, eructation, however, these patients were did not diagnosed as serious GI complications.

Additionally, Asymptomatic GI injury was not considered in this study due to patients did not experience any peptic symptoms.

\subsection{Data Collection}

Patient data were collected through CRF, which was approved in advance by the Independent Ethics Committee. The collected information included patient gender and age, smoking and drinking habits, and past history and medications. Conditions noted in the CRF included hypertension, diabetes, hyperlipidemia, stroke, and renal insufficiency. Medications recorded and monitored during hospitalization included antiplatelet agents and combined medication such as gastroprotective agents (proton pump inhibitor [PPI]), anticoagulant agents (heparin sodium, dabigatran, warfarin sodium), the antiplatelet agent ticagrelor, vasodilator drugs ( $\beta$-blocker, calcium channel blocker [CCB], angiotensin converting enzyme inhibitors [ACEI], angiotensin receptor blocker [ARB], diuretics), antidiabetics, the cardiotonic drug digoxin, and the antiarrhythmic drug amiodaron.

During follow-up, GI injury from hospital readmission records provided by the referring physician or the outpatient clinic, and reported by the patient in the phone interview, were included in the computer database.

\subsection{Statistical Analysis}

Continuous variables were expressed as frequency and percentages, while quantitative data was expressed as mean \pm standard deviation. Because the sample size is 603 that it can be regard as a normal distribution. Differences in gender, risk factors, concomitant medications, and clinical outcomes between serious GI complications and non-serious GI complications groups were evaluated using chi-squared tests, while Student's t-test was used to assess differences for continuous variables. Cox regression analysis was used to assess the risk factors of serious GI complications, expressed as a hazard ratio (HR) with a $95 \%$ confidence interval (CI). Statistical analysis of all data was performed by IBM SPSS Statistics 22.0 software. $\mathrm{P}<0.05$ was considered statistically significant.

\section{Result}

\subsection{Characteristics of the Study Population}

603 eligible patients were enrolled, with age $(65.75 \pm 11.02)$ [male: $338(56.1 \%)$ 
with age $70.67 \pm 8.62$, female: 265 (43.9\%) with age $65.03 \pm 11.90$ ]. In these patients (see Table 1), 148 (24.5\%) patients were smokers, and 62 (10.3\%) consumed alcohol. Regarding past history, 403 (66.8\%) had hypertension, 120 (19.9\%) had diabetes, 119 (19.7\%) had experienced a stroke, 64 (10.6\%) had GI injury, 59 (9.8\%) had hyperlipidemia, and $7(1.2 \%)$ had renal insufficiency. For the concomitant medication, 421 (69.8\%) $\beta$-blocker, 404 (67.0\%) received PPI at the hospital, 265 (43.9\%) CCB, 250 (41.5\%) heparin sodium, 197 (32.7\%) ARB, $110(18.2 \%)$ ACEI, 82 (13.6\%) diuretics, 71 (11.8\%) antidiabetics, 33 (5.5\%) ticagrelor, $21(3.5 \%)$ warfarin, $14(2.3 \%)$ digoxin, $14(2.3 \%)$ amiodarone and 6 (1.0\%) dabigatran.

Table 1. Demographics characteristic of whole study cohort.

\begin{tabular}{|c|c|}
\hline Parameter & Total $(\mathrm{N}=603)$ \\
\hline Gender, $\mathrm{n}$ (\% male) & $338(56.1 \%)$ \\
\hline Age, year & $65.75 \pm 11.02$ \\
\hline Smoking, n (\%) & $148(24.5 \%)$ \\
\hline Drinking, n (\%) & $62(10.3 \%)$ \\
\hline \multicolumn{2}{|l|}{ Past history: } \\
\hline Hypertension, n (\%) & $403(66.8 \%)$ \\
\hline Diabetes, n (\%) & $120(19.9 \%)$ \\
\hline Hyperlipidemia, n (\%) & $59(9.8 \%)$ \\
\hline Stroke, n (\%) & $119(19.7 \%)$ \\
\hline Renal insufficiency, $\mathrm{n}(\%)$ & $7(1.2 \%)$ \\
\hline Peptic injury, n (\%) & $64(10.6 \%)$ \\
\hline \multicolumn{2}{|l|}{ Combined medication: } \\
\hline PPI (\%) & $404(67.0 \%)$ \\
\hline Heparin sodium, $\mathrm{n}(\%)$ & $250(41.5 \%)$ \\
\hline Dabigatran, n (\%) & $6(1.0 \%)$ \\
\hline Warfarin, n (\%) & $21(3.5 \%)$ \\
\hline Ticagrelor, n (\%) & $33(5.5 \%)$ \\
\hline$\beta$-blocker, n (\%) & $421(69.8 \%)$ \\
\hline CCB, n (\%) & $265(43.9 \%)$ \\
\hline ACEI, $n(\%)$ & $110(18.2 \%)$ \\
\hline $\mathrm{ARB}, \mathrm{n}(\%)$ & $197(32.7 \%)$ \\
\hline Diuretics, n (\%) & $82(13.6 \%)$ \\
\hline Antidiabetics, n (\%) & $71(11.8 \%)$ \\
\hline Digoxin, n (\%) & $14(2.3 \%)$ \\
\hline Amiodarone, n (\%) & $14(2.3 \%)$ \\
\hline
\end{tabular}

PPI: proton pump inhibitor; CCB: calcium channel blocker; ACEI: angiotensin converting enzyme inhibitors; ARB: angiotensin receptor blocker. 


\subsection{Incidence of Serious GI Complications and GI Symptoms}

After one year's follow-up, the total incidence of symptomatic GI injury was 17.9\%: $3.65 \%$ of serious GI complications, and $14.26 \%$ of GI symptoms. In order to identify the high-frequency periods of GI injury, the incidences during four periods were calculated (Table 2). The incidences of serious GI complications during $0-1,1-3,3-6$, and $6-12$ months were $0.17 \%, 2.82 \%, 0.17 \%$, and $0.50 \%$ respectively. A similar trend was evident for GI symptoms, at 3.15\%, 6.96\%, $1.99 \%$, and $2.16 \%$. All symptomatic GI injuries in total were $3.32 \%, 9.78 \%$, $2.16 \%$, and $2.66 \%$.

\subsection{Cox Regression Analysis of Serious GI Complications}

In this study, we focused on the risk factors of serious GI complications, which cause more serious consequences. After one year of follow-up, a total of 22 (3.65\%) out of 603 patients developed serious GI complications, and another 581 (96.35\%) had not (Table 3). Comparing two groups, the proportion of smoking [45.4\% vs. $23.8 \%$ (serious vs. non-serious), $\mathrm{P}=0.020]$ and drinking $(22.7 \%$ vs. $9.8 \%, \mathrm{P}=0.049$ ) in patients with serious GI complications was significantly higher. The serious GI complications group also had a higher rate of previous peptic injury (27.3\% vs. $10.0 \%, \mathrm{P}=0.026)$, and less frequent use of PPI $(45.5 \%$ vs. $67.8 \%, \mathrm{P}=0.029)$ after DAPT. There were no significant differences between the two groups with regard to gender or age, or history of hypertension, diabetes, hyperlipidemia, stroke, renal insufficiency, or concomitant drugs.

To further analyze the risk factors of serious GI complications, a cox multivariate regression analysis was used with forward conditional Wald variant (Table 4). The risks factors of age ( $>75$ years), smoking, drinking, previous peptic injury, and use of PPI were enrolled into the cox regression model. Cox multivariate regression analysis shows that the risk factors of drinking $(\mathrm{HR}=3.647 ; 95 \%$ CI $[1.512,8.796] ; \mathrm{P}=0.004)$ and previous peptic injury $(\mathrm{HR}=6.458 ; 95 \% \mathrm{CI}$ $[2.307,18.080] ; \mathrm{P}=0.001$ ) were independent risk factors for serious GI complications in patients with ACS receiving DAPT, while use of PPIs $(\mathrm{HR}=0.290$; $95 \%$ CI $[0.12,0.699] ; \mathrm{P}=0.006)$ was a protective factor in these patients. Of these influence factors, previous peptic injury was the most important risk factor, and the probability of serious GI complications was 6.458 times of that in patients who without. The total incidence of serious GI complications at one-year follow-up is shown in Figure 2.

Table 2. Incidences of serious GI complications and GI symptoms during certain periods.

\begin{tabular}{cccccc}
\hline periods & $0-1$ month & $1-3$ month & $3-6$ month & $6-12$ month & Total \\
\hline serious GI complications & $0.166 \%$ & $2.82 \%$ & $0.166 \%$ & $0.498 \%$ & $3.65 \%$ \\
GI symptoms & $3.15 \%$ & $6.96 \%$ & $1.99 \%$ & $2.16 \%$ & $14.26 \%$ \\
symptomatic GI injury & $3.32 \%$ & $9.78 \%$ & $2.16 \%$ & $2.66 \%$ & $17.9 \%$ \\
\hline
\end{tabular}

GI: gastrointestinal. 
Table 3. Demographic characteristics of patients between serious GI complications and non-serious GI complications group.

\begin{tabular}{|c|c|c|c|}
\hline Parameters & serious $(\mathrm{N}=22)$ & non-serious $(\mathrm{N}=581)$ & $P$ value \\
\hline Gender, $\mathrm{n}$ (\% male) & $15(68.2 \%)$ & $323(55.6 \%)$ & 0.243 \\
\hline Age, year & $70.09 \pm 8.59$ & $65.59 \pm 11.08$ & 0.060 \\
\hline Smoking, n (\%) & $10(45.4 \%)$ & $138(23.8 \%)$ & 0.020 \\
\hline Drinking, n (\%) & $5(22.7 \%)$ & $57(9.8 \%)$ & 0.049 \\
\hline \multicolumn{4}{|l|}{ Past history: } \\
\hline Hypertension, n (\%) & $14(63.6 \%)$ & $389(67.0 \%)$ & 0.746 \\
\hline Diabetes, n (\%) & $8(36.4 \%)$ & $112(19.3 \%)$ & 0.089 \\
\hline Hyperlipidemia, n (\%) & $1(4.5 \%)$ & $58(10.0 \%)$ & 0.633 \\
\hline Stroke, n (\%) & $3(13.6 \%)$ & $116(20.0 \%)$ & 0.646 \\
\hline Renal insufficiency, n (\%) & $0(0 \%)$ & $7(1.2 \%)$ & 1.000 \\
\hline Peptic injury, n (\%) & $6(27.3 \%)$ & $58(10.0 \%)$ & 0.026 \\
\hline \multicolumn{4}{|l|}{ Combined medication: } \\
\hline PPI (\%) & $10(45.5 \%)$ & $394(67.8 \%)$ & 0.029 \\
\hline Heparin sodium, n (\%) & $13(59.1 \%)$ & $237(40.8 \%)$ & 0.087 \\
\hline Dabigatran, n (\%) & $0(0 \%)$ & $6(1.0 \%)$ & 1.000 \\
\hline Warfarin, n (\%) & $0(0 \%)$ & $21(3.6 \%)$ & 1.000 \\
\hline Ticagrelor, n (\%) & $2(9.1 \%)$ & $31(5.3 \%)$ & 0.777 \\
\hline$\beta$-blocker, $\mathrm{n}(\%)$ & $14(63.6 \%)$ & $407(70.1 \%)$ & 0.520 \\
\hline $\mathrm{CCB}, \mathrm{n}(\%)$ & $11(50.0 \%)$ & $254(43.7 \%)$ & 0.560 \\
\hline ACEI, n (\%) & $4(18.2 \%)$ & $106(18.2 \%)$ & 1.000 \\
\hline $\mathrm{ARB}, \mathrm{n}(\%)$ & $7(31.8 \%)$ & $190(32.7 \%)$ & 0.931 \\
\hline Diuretics, n (\%) & $2(9.1 \%)$ & $80(13.8 \%)$ & 0.755 \\
\hline Antidiabetics, n (\%) & $5(22.7 \%)$ & $66(11.4 \%)$ & 0.198 \\
\hline Digoxin, n (\%) & $0(0 \%)$ & $14(2.4 \%)$ & 1.000 \\
\hline Amiodarone, n (\%) & $0(0 \%)$ & $14(2.4 \%)$ & 1.000 \\
\hline
\end{tabular}

PPI: proton pump inhibitor; CCB: calcium channel blocker; ACEI: angiotensin converting enzyme inhibitors; ARB: angiotensin receptor blocker.

Table 4. Independent predictors of serious GI complications in one year.

\begin{tabular}{ccccc}
\hline \multirow{2}{*}{ Risk factors } & Hazard ratio & \multicolumn{2}{c}{$95.0 \%$ CI } & \multirow{2}{*}{ P value } \\
\cline { 3 - 4 } & & Lower & Upper & \\
\hline Drinking habit & 3.647 & 1.512 & 8.796 & 0.004 \\
Previous peptic injury & 6.458 & 2.307 & 18.08 & 0.001 \\
Using PPIs & 0.29 & 0.12 & 0.699 & 0.006 \\
\hline
\end{tabular}

PPIs: proton pump inhibitors. 


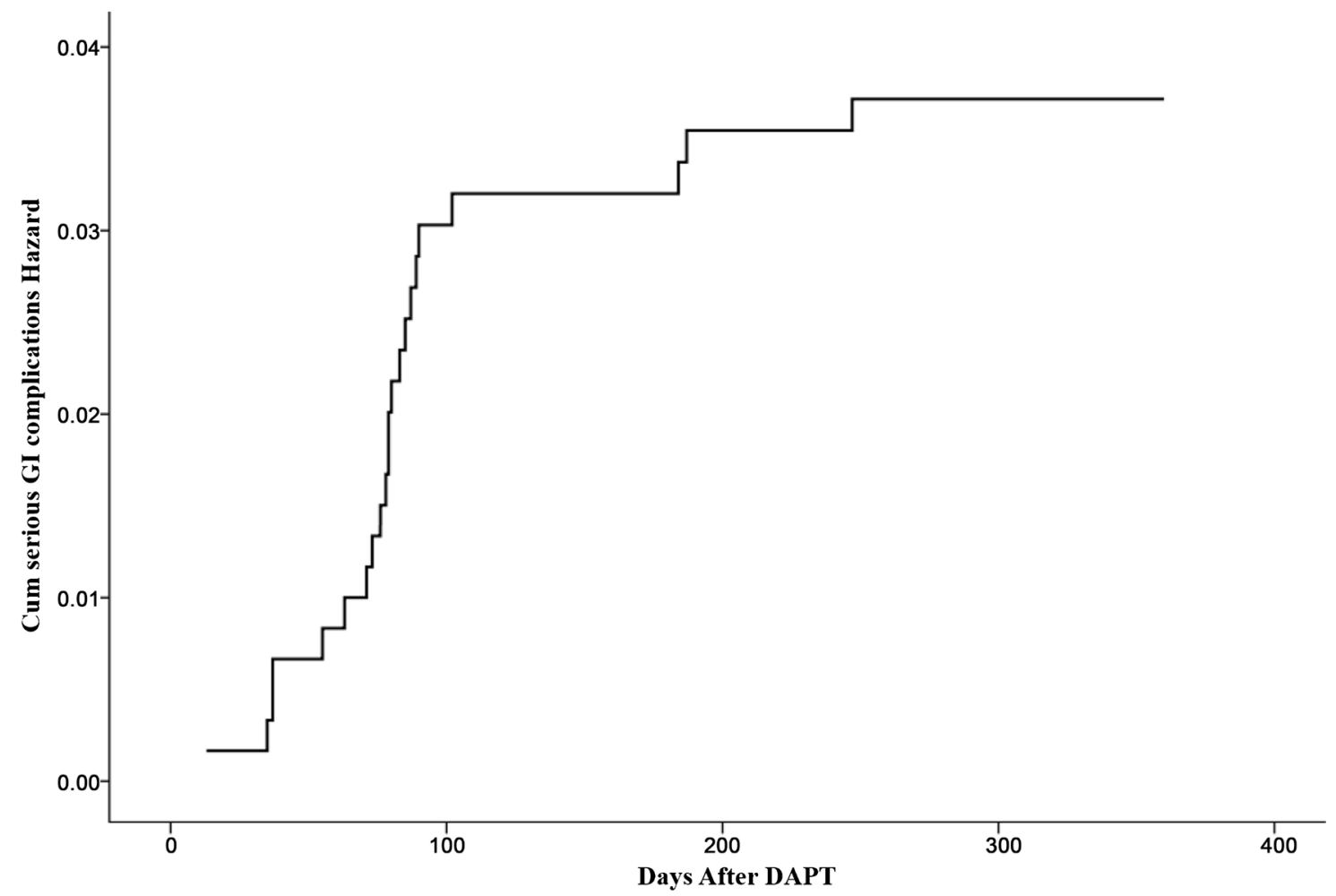

Figure 2. The risk curve of serious GI complications.

\section{Discussion}

In this study, we found that the incidence of total symptomatic GI injury within one-year follow-up was $17.9 \%$, including $3.65 \%$ of serious GI complications and $14.2 \%$ of GI symptoms. This suggests that symptomatic GI injury is relatively common in ACS patients with DAPT. Meanwhile, the incidences of both serious GI complications and GI symptoms increased in the first three months after DAPT. Additionally, we found that previous peptic injury and drinking habit were independent risk factors for serious GI complications, while the use of PPIs was a protective factor in these patients.

Several studies describe the incidence of GI injury in patients taking DAPT. The incidence of GI bleeding was $2.7 \%$ during the DAPT therapy or within 7 days of stopping enoxaparin in NG's study [11]. In another study, $1.2 \%$ of patients developed UGIB in the 30 days following PCI [12]. In our study, the occurrence of serious GI complications was 3.65\%, which is higher than in the other studies [9] [11] [12]. One reason for this is that serious GI complications in our study included GI ulcers, bleeding, perforation, etc. And another is that we conducted a one-year follow-up, which is much longer than the 7 or 30 days in these two studies.

An important attribute of our study is the analysis of the occurrence of symptomatic GI injury in ACS patients with DAPT. To the best of our knowledge, this is the first report on the incidence of symptomatic GI injury in ACS patients who received DAPT after one year's follow-up. It is important to pay attention 
to symptomatic GI injury in ACS patients because it is a common reason for discontinuation of antiplatelet treatments [14]. A recent study [15] described that one-third of patients had discontinued at least one of their prescribed treatments in three months after acute coronary syndrome, low dose aspirin accounting for $6.7 \%$ of all discontinuation. Poor aspirin compliance might be critical in high-risk vascular patients or stented patients treated with DAPT, especially when clopidogrel is discontinued leaving patients without any effective antiplatelet therapy and exposed to acute complications like stent thrombosis [16] [17] [18]. Our study shows that symptomatic GI injury is present in one-sixth of ACS patients, which suggests that physicians should pay attention to these patients to improve symptoms and prevent inadequate interruption in high-risk vascular patients.

The findings are therefore that the proportion of both serious GI complications and GI symptoms increased most rapidly in the first three months, suggesting that the first three months have the highest risk of symptomatic GI injury. This phenomenon has been explained as a consequence of gastric adaptation to DAPT [19] or a fall in the proportion of susceptible individuals due to treatment withdrawal following GI intolerance, peptic complication, or other adverse effects [20] in later follow-up. Regardless, this result indicates that the first three months after DAPT is the higher-risk period, which necessitates intervention and review.

Our study showed that previous peptic injury and drinking habit increased patients' risk of serious GI complications. Previous peptic injury is an independent risk factor, which is consistent with relevant studies [21] [22]. In our study, HR for the factor of previous peptic injury was 6.458, indicating a higher risk; other research reported HR of 3.270 and 4.155 [23] [24]. It can be explained that previous studies focused on simple GI bleeding, while the end points of ulcer, pyloric obstruction, perforation, and death are included in ours, which led to a higher incidence in our study. Additionally, types of previous GI disease were different, offering limited comparability. However, the conclusion can still be drawn that previous peptic injury was the highest risk factor of serious GI complications in ACS patients receiving DAPT. This might be because previous peptic injury can weaken the defenses of the digestive tract, resulting in more sensitivity to antiplatelet drugs and stressful injury of ACS disease. Drinking habit was another risk factor in our study, and the relationship between drinking and UGIB has been previous demonstrated [25] [26], although some other studies [24] [27] have indicated that drinking might not increase the risk of GI bleeding. The diversity observed between our study and previous studies could be due to different patient populations, different factors included, different end points, and varying follow-up periods.

In several studies, advanced age was also an independent risk factor for GI bleeding in PCI patients [24] [28]. However, in other studies and ours, age was not the primary risk factors of serious GI complications for ACS with DAPT [21] [22]. This might be because the mean age of patients in these studies was 
younger; the tolerance for GI illness in younger patients is often better [19] [20]. Additionally, the mean of age in our study was about 65 years, indicating the result in our study is essentially the same as another study [20] which reported that age of $>65$ years, and especially $>70$, had only moderate risk.

Regarding the protective factor, our study suggests that using PPIs is a protective factor for serious GI complications in ACS patients with DAPT. Cox regression analysis adjusting for age, drinking and smoking, and previous peptic injury finds that PPI treatment is an effective prevention against serious GI complications. The result is consistent with several studies [11] [23], which found that co-prescription with PPI can significantly reduce the risk of GI bleeding. It might because that PPI inhibits gastric acid secretion and thus reduces the PH value of the stomach to prevent mucosal damage and bleeding and other complications induced by DAPT [29]. Therefore, in patients with moderate risk, physicians should consider individualized risk assessment when prescribing drugs or performing procedures that might increase the risk of GI injury, and take necessary measures to reduce modifiable risk factors such as lifestyle counseling or using PPIs in the first three months after DAPT.

The strength of this study is that it was a large hospital-based prospective survey, with a high rate of response. The data comes from real world, serves practical purpose to guide clinical application. Some limitations of our research should be considered. First this study didn't provide information about the prevalence of GI injury in ACS patients who did not use DAPT as a contrast. Second, other factors reported by previous surveys may influence the GI injury [30] [31], such as H. pylori infection. However, we didn't evaluate H. pylori infection due to limited funds and $H$. pylori was not routinely tested for by cardiologists in our hospital. Third, our study did not investigate how symptomatic GI injury affects patients' compliance to DAPT and cardiovascular events. Therefore, A randomized controlled and multicenter study with a larger cohort of patients is needed in the future so that the risk factors and protective factors can be defined more precisely.

\section{Conclusion}

In conclusion, Symptomatic GI injuries are relatively common in ACS patients with DAPT in one year, with the incidence of total GI injury at $17.9 \%$, including $3.65 \%$ of serious GI complications and $14.2 \%$ of GI symptoms, all peaking in the first three months. Besides, previous peptic injury and drinking habit were independent risk factors for serious GI complications, while use of PPIs was a protective factor in these patients. Those mean that PPI can be used for ACS patients with DAPT to prevent GI injury in the first three months in clinical, especially for people who lives with high risk.

\section{Acknowledgements}

We are grateful to all members of the Division of Cardiology and Pharmacy, 
Zhongda Hospital, Southeast University, for their help in this study. This research was supported by the Natural Science Foundation of China (No. 81700322), the Nanjing Pharmaceutical Association-Changzhou Siyao Pharmaceuticals Co., Ltd Hospital Pharmacy Research fund (2014YX009) and Jiangsu Province Pharmaceutical Association-Baxter Biopharmaceutical Co., Ltd Hospital Pharmacy Research Fund (2014JSSYXH004).

\section{Conflicts of Interest}

No potential conflict of interest is relevant to this article.

\section{References}

[1] Curial, M., Nath, E. and Lang, E. (2013) Novel Antiplatelet Agent Use for Acute Coronary Syndrome in the Emergency Department: A Review. Cardiology Research and Practice, 2013, Article ID: 127270.

[2] Vengoechea, F. (2014) Management of Acute Coronary Syndrome in the Hospital: A Focus on ACCF/AHA Guideline Updates to Oral Antiplatelet Therapy. Hospital Practice, 42, 33-47. https://doi.org/10.3810/hp.2014.08.1116

[3] Yahya, D., Salar, S. and Javad, K. (2016) Risks and Benefits of Dual Antiplatelet Therapy beyond 12 Months After Coronary Stenting: A Prospective Randomized Cohort Study. Medicine, 95, e3663. https://doi.org/10.1097/MD.0000000000003663

[4] Briasoulis, A. and Palla, M. (2016) Duration of Dual Antiplatelet Therapy after Coronary Stenting. International Journal of Cardiology, 222, 1064-1067. https://doi.org/10.1016/j.ijcard.2015.10.101

[5] Bobadilla, R.V. (2016) Acute Coronary Syndrome: Focus on Antiplatelet Therapy. Critical Care Nurse, 36, 15-27. https://doi.org/10.4037/ccn2016497

[6] Liu. Y., Liu, N., Li, W., et al. (2011) Clopidogrel Response Variability and Its Correlation with Early Recurrent Cardiovascular Events in Chinese Patients Undergoing Percutaneous Coronary Intervention. Pharmacology, 87, 321-330. https://doi.org/10.1159/000324529

[7] Damman, P., van't Hof, A.W., Ten, J.B., et al. (2017) 2015 ESC Guidelines for the Management of Acute Coronary Syndromes in Patients Presenting without Persistent ST-Segment Elevation: Comments from the Dutch ACS Working Group. Netherlands Heart Journal, 25, 181-185. https://doi.org/10.1007/s12471-016-0939-y

[8] Levine, G.N., Bates, E.R. and Blankenship, J.C. (2011) 2011 ACCF/AHA/SCAI Guideline for Percutaneous Coronary Intervention: A Report of the American College of Cardiology Foundation/American Heart Association Task Force on Practice Guidelines and the Society for Cardiovascular Angiography and Interventions. Journal of the American College of Cardiology, 58, 44-122. https://doi.org/10.1016/j.jacc.2011.08.007

[9] Lanas, A., Wu, P., Medin, J., et al. (2011) Low Doses of Acetylsalicylic Acid Increase risk of Gastrointestinal Bleeding in A Meta-Analysis. Clinical Gastroenterology \& Hepatology, 9, 762-768. https://doi.org/10.1016/j.cgh.2011.05.020

[10] Sesler, J.M. (2007) Stress-Related Mucosal Disease in the Intensive Care Unit: An Update on Prophylaxis. AACN Advanced Critical Care, 18, 119-126. https://doi.org/10.1097/01.AACN.0000269254.39967.8e

[11] Ng, F.H., Wong, S.Y., Lam, K.F., et al. (2008) Gastrointestinal Bleeding in Patients Receiving a Combination of Aspirin, Clopidogrel, and Enoxaparin in Acute Coro- 
nary Syndrome. The American Journal of Gastroenterology, 103, 865-871. https://doi.org/10.1111/j.1572-0241.2007.01715.x

[12] Chin, M. (2007) Predictive and Protective Factors Associated with Upper Gastrointestinal Bleeding after Percutaneous Coronary Intervension: A Case-Control Study. American Journal of Gastroenterology, 102, 2441-2416.

[13] Brown, T.J., Hooper, L., Elliott, R.A., et al. (2006) A Comparison of the Cost-Effectiveness of Five Strategies for the Prevention of Non-Steroidal Anti-Inflammatory Drug-Induced Gastrointestinal Toxicity: A Systematic Review with Economic Modelling-NIHR Health Technology Assessment Programme: Executive Sum. Nihr Evaluation Trials \& Studies Coordinating Centre.

[14] Biondizoccai, G.G., Lotrionte, M., Agostoni, P., et al. (2006) A Systematic Review and Meta-Analysis on the Hazards of Discontinuing or Not Adhering to Aspirin among 50,279 Patients at Risk for Coronary Artery Disease. European Heart Journal, 27, 2667-2674. https://doi.org/10.1093/eurheartj/ehl334

[15] Melloni, C., Alexander, K.P., Ou, F.S., et al. (2009) Predictors of Early Discontinuation of Evidence-Based Medicine after Acute Coronary Syndrome. American Journal of Cardiology, 104, 175-181. https://doi.org/10.1016/j.amjcard.2009.03.013

[16] Grant, C. and Veal, A.J. (2012) Prevalence and Clinical Impact of Upper Gastrointestinal Symptoms in Subjects Treated with Low Dose Aspirin: The UGLA Survey. International Journal of Cardiology, 156, 69-75. https://doi.org/10.1016/j.ijcard.2010.10.027

[17] Collet, J.P., Montalescot, G., Blanchet, B., et al. (2004) Impact of Prior Use or Recent Withdrawal of Oral Antiplatelet Agents on Acute Coronary Syndromes. Circulation, 110, 2361-2367. https://doi.org/10.1161/01.CIR.0000145171.89690.B4

[18] Eisenberg, M.J., Richard, P.R., Libersan, D., et al. (2009) Safety of Short-Term Discontinuation of Antiplatelet Therapy in Patients with Drug-Eluting Stents. Circulation, 119, 1634-1642. https://doi.org/10.1161/CIRCULATIONAHA.108.813667

[19] Graham, D.Y., Smith, J.L., Spjut, H.J., et al. (1988) Gastric Adaptation. Studies in Humans during Continuous Aspirin Administration. Gastroenterology, 95, 327-333. https://doi.org/10.1016/0016-5085(88)90487-8

[20] Baigent, C., Blackwell, L., Collins, R., et al. (2009) Aspirin in the Primary and Secondary Prevention of Vascular Disease: Collaborative Meta-Analysis of Individual Participant Data from Randomized Trial Antithrombotic Trialists' (ATT) Collaboration. The Lancet, 373, 1849-1860.

[21] Lee, J.M., Park, S.Y., Choi, J.H., et al. (2016) Clinical Risk Factors for Upper Gastrointestinal Bleeding after Percutaneous Coronary Intervention: A Single-Center Study. Gut \& Liver, 10, 58-62. https://doi.org/10.5009/gnl14127

[22] Jensen, B.E., Hansen, J.M., Junker, A.B., et al. (2015) High Prevalence of Ulcer Bleeding Risk Factors in Dual Antiplatelet-Treated Patients after Percutaneous Coronary Intervention. Danish Medical Journal, 62, A5092.

[23] Jiang, Z., Wu, H., Duanet, Z., et al. (2013) Proton-Pump Inhibitors Can Decrease Gastrointestinal Bleeding after Percutaneous Coronary Intervention. Clinics and Research in Hepatology and Gastroenterology, 37, 636-641. https://doi.org/10.1016/j.clinre.2013.03.011

[24] Huang, K.W., Luo, J.C., Leu, H.B., et al. (2013) Risk Factors for Upper Gastrointestinal Bleeding in Coronary Artery Disease Patients Receiving both Aspirin and Clopidogrel. Journal of the Chinese Medical Association, 76, 9-14. https://doi.org/10.1016/j.jcma.2012.09.004

[25] Watanabe, H., Kamijima, Y., Sato, T., et al. (2009) Ex-Drinking May Be a Surrogate 
for Unmeasured Risk Factors for Upper Gastrointestinal Bleeding: Reappraisal and an Additional Survey of Subjects from a Case-Control Study in Japan. European Journal of Epidemiology, 24, 143-147. https://doi.org/10.1007/s10654-009-9319-x

[26] Kaufman, D.W., Kelly, J.P., Wiholm, B.E., et al. (1999) The Risk of Acute Major Upper Gastrointestinal Bleeding among Users of Aspirin and Ibuprofen at Various Levels of Alcohol Consumption. The American Journal of Gastroenterology, 94, 3189-3196. https://doi.org/10.1111/j.1572-0241.1999.01517.x

[27] Vaduganathan, M., Bhatt, D.L., Cryer, B.L., et al. (2016) Proton-Pump Inhibitors Reduce Gastrointestinal Events Regardless of Aspirin Dose in Patients Requiring Dual Antiplatelet Therapy. Journal of the American College of Cardiology, 67, 1661-1671. https://doi.org/10.1016/j.jacc.2015.12.068

[28] Nadatani, Y., Watanabe, T., Tanigawa, T., et al. (2013) Incidence and Risk Factors of Gastrointestinal Bleeding in Patients on Low-Dose Aspirin Therapy after Percutaneous Coronary Intervention in Japan. Scandinavian Journal of Gastroenterology, 48, 320-325. https://doi.org/10.3109/00365521.2012.758771

[29] Schubert, M.L. (2017) Physiologic, Pathophysiologic, and Pharmacologic Regulation of Gastric Acid Secretion. Current Opinion in Gastroenterology, 33, 430-438. https://doi.org/10.1097/MOG.0000000000000392

[30] Yakabi, K., Yamaguchi, N., Ono, S., et al. (2017) Open Label Trial of the Efficacy and Safety Profile of Rikkunshito Used for the Treatment of Gastrointestinal Symptoms in Patients with Parkinson's Disease: A Pilot Study. Current Therapeutic Research, 87, 1-8. https://doi.org/10.1016/j.curtheres.2017.07.003

[31] Lanas, A. (2001) Preventing Recurrent Upper Gastrointestinal Bleeding in Patients with Helicobacter Pylori Infection Who Are Taking Low-Dose Aspirin or Naproxen -NEJM. The New England Journal of Medicine, 344, 967-973. https://doi.org/10.1056/NEJM200103293441304

\section{List of Abbreviations}

GI: gastrointestinal

ACS: acute coronary syndrome

DAPT: double antiplatelet therapy

PPI: proton pump inhibitor

STEMI: ST segment elevation myocardial infarction

NSTEMI: non-ST segment elevation myocardial infarction

UA: unstable angina

CRF: case report form

UGIB: upper GI bleeding

CCB: calcium channel blocker

ACEI: angiotensin converting enzyme inhibitors

ARB: angiotensin receptor blocker

HR: hazard ratio

95\% CI: confidence interval. 


\section{CRF}

Inclusion criteria:

Age $\geq 18$

Platelet count: $100 \sim 350 \times 10^{9} / \mathrm{L}$

Hemoglobin $\geq 10 \mathrm{~g} / \mathrm{dl}$

Diagnosed as ACS

Receiving dual antiplatelet therapy

Did not take proton pump inhibitor

Exclusion criteria:

Severe bleeding disease within 1 month

Combined with other serious organ diseases

Used glucocorticoids within 1 month

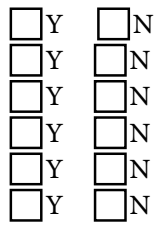

\begin{tabular}{llll}
\hline & & \multicolumn{2}{c}{ Basic information } \\
\hline Name: Gender: & $\square$ male $\square$ female & Date of birth: & Hospital number: \\
Native place: & Race: & Working place: & Telephone: \\
Height: $(\mathrm{cm})$ & Weigh: $(\mathrm{kg})$ & BMI: $\left(\mathrm{kg} / \mathrm{m}^{2}\right)$ & \\
Admission time: & & Discharge time: &
\end{tabular}

Past medical history

Bad hobby

Smoke(Y, N),

cigarettes /day

$\operatorname{Drink}(\mathrm{Y}, \mathrm{N}), \mathrm{ml} /$ day

Allergy history:

Drug allergy history

Past medication history:

Drug name:

$\square \mathrm{Y} \square \mathrm{N}$

$\square \mathrm{Y} \square \mathrm{N}$

$\square \mathrm{Y} \square \mathrm{N}$

Basic informatio

History of other common disease:

Hypertension

Diabetes

Heart failure

Cerebrovascular event

Head injury

Chronic obstructive pulmonary disease

Chronic renal insufficiency

Gastrointestinal Diseases

Hyperthyroidism

Hematopathy

Organ transplant

Receiving glucocorticoid

Receiving immunosuppressive therapy

Family history:

Hypertension

Diabetes

Coronary disease

Other diseases

Clinical efficacy

\begin{tabular}{|c|c|c|c|c|c|c|}
\hline Clinical efficacy & & & & \multirow[t]{2}{*}{ Occurrence time } & \multicolumn{2}{|l|}{ Treatment } \\
\hline \multirow{3}{*}{$\begin{array}{l}\text { Clinical efficacy } \\
\text { evaluation index }\end{array}$} & cardiovascular death & $7 \mathrm{~N}$ & $\overline{\mathrm{Y}}$ & & & \\
\hline & Acute and subacute stent thrombosis & $7 \mathrm{~N}$ & ] $\mathrm{Y}$ & & & \\
\hline & Relapse ACS & $7 \mathrm{~N}$ & YY & & & \\
\hline \multicolumn{4}{|c|}{ Gastrointestinal injury } & Occurrence time & Duration & Treatment \\
\hline \multirow{6}{*}{$\begin{array}{l}\text { Gastrointestinal } \\
\text { symptoms }\end{array}$} & Nausea & $7 \mathrm{~N}$ & ] $\mathrm{Y}$ & & & \\
\hline & Vomit & $7 \mathrm{~N}$ & $\overline{\mathrm{Y}}$ & & & \\
\hline & Abdominal pain & $\overline{\mathrm{N}}$ & ] $\mathrm{Y}$ & & & \\
\hline & Diarrhea & $7 \mathrm{~N}$ & $7 \mathrm{Y}$ & & & \\
\hline & Haematemesis & $7 \mathrm{~N}$ & $\overline{\mathrm{Y}}$ & & & \\
\hline & Melena & $7 \mathrm{~N}$ & ] $\mathrm{Y}$ & & & \\
\hline \multirow[t]{3}{*}{ Diagnosis } & Gastrointestinal mucosal erosion & $7 \mathrm{~N}$ & ]Y & & & \\
\hline & Gastrointestinal ulcer & $7 \mathrm{~N}$ & ] $\mathrm{Y}$ & & & \\
\hline & Gastrointestinal bleeding & $\square \mathrm{N}$ & ]Y & & & \\
\hline \multicolumn{2}{|l|}{ Adverse reactions: } & $7 \mathrm{~N}$ & $\overline{\mathrm{Y}}$ & & & \\
\hline
\end{tabular}

